\title{
过氧自由基化学放大测定方法中的水效应：实验 测定及其化学机制
}

齐斌"，刘路，昆余涛，王竹青，杨红艳

陕西师范大学化学与材料科学学院, 西安 710062

*联系人, E-mail: b.qi@163.com

收稿日期: 2007-06-21; 接受日期: 2007-08-02

摘要 在室温 $(298 \pm 2) \mathrm{K}$ 、大气压 $\left(1 \times 10^{5} \mathrm{~Pa}\right)$ 下, 分别测定了 $\mathrm{HO}_{2}$ 及 $\mathrm{HO}_{2}+\mathrm{OH}$ 混合自由基在化 学放大过程中的水效应, 发现水效应不随自由基的类型有显著变化. 在此基础上, 采用密度泛 函理论在 $\mathrm{CCSD}(\mathrm{T}) / 6-311 \mathrm{G}(2 \mathrm{~d}, 2 \mathrm{p}) / / \mathrm{B} 3 \mathrm{LYP} / 6-311 \mathrm{G}(2 \mathrm{~d}, 2 \mathrm{P})$ 水平上研究了 $\mathrm{HO}_{2}$ 自由基-水加合物 $\mathrm{HO}_{2} \cdot \mathrm{H}_{2} \mathrm{O}$ 与 $\mathrm{NO}$ 的反应. 计算结果表明 $\mathrm{HO}_{2} \cdot \mathrm{H}_{2} \mathrm{O}$ 与 $\mathrm{NO}$ 主反应通道为 $\mathrm{HO}_{2} \cdot \mathrm{H}_{2} \mathrm{O}+\mathrm{NO} \rightarrow \mathrm{HNO}_{3}$ $+\mathrm{H}_{2} \mathrm{O}$ (R4a). 应用 Polyrate 程序计算了(R4a)的速率常数. 在 200 2000 K 温度范围内, 拟合得 到该反应速率常数的三参数 Arrenhnius 方程为: $k=5.49 \times 10^{7} T^{1.03} \exp (-14798 / T)$. 将理论计算

关键词 过氧自由基 化学放大 水效应 机制 密度泛函理论 结果用于模式计算中模拟得到的水效应曲线与实验测定曲线一致, 表明(R4a)可能是形成水效 应的主要原因。

过氧自由基化学放大测定方法(Peroxy Radical Measurement by Chemical Amplification, PERCA)灵 敏度高, 响应快, 能实时、在线、连续地测定大气过 氧自由基，同时体积小，质量轻，因而被广泛应用于 大气外场观测 ${ }^{[1,2]}$. PERCA测定过氧自由基的原理是 在过量 $\mathrm{NO}$ 和 $\mathrm{CO}$ 存在下, 通过链反应将 $\mathrm{NO}$ 催化氧化 为大量的 $\mathrm{NO}_{2}{ }^{[3]}$. 以 $\mathrm{HO}_{2}$ 自由基为例, 化学放大过程 如下:

$$
\begin{gathered}
\mathrm{HO}_{2}+\mathrm{NO} \rightarrow \mathrm{NO}_{2}+\mathrm{OH} \\
\mathrm{OH}+\mathrm{CO} \rightarrow \mathrm{H}+\mathrm{CO}_{2} \\
\mathrm{H}+\mathrm{O}_{2}+\mathrm{M} \rightarrow \mathrm{HO}_{2}+\mathrm{M}
\end{gathered}
$$

通过测定链反应长度即链长 $(\mathrm{CL})$ 及化学放大生成的 $\mathrm{NO}_{2}$ 的浓度, 即可以计算出自由基的浓度: $\left[\mathrm{HO}_{2}\right]=$ $\left[\mathrm{NO}_{2}\right] / \mathrm{CL}$. 通常气体样品中还存在除化学放大外其 他来源的 $\mathrm{NO}_{2}$. 这部分 $\mathrm{NO}_{2}$ 需要从测定结果中扣除. 这可以通过调制技术完成, 即周期性地将 $\mathrm{CO}$ 加入位
置从化学放大器的入口移到出口处, 使化学放大不 断处于开启和闭合状态 ${ }^{[4]}$, 自由基浓度则正比于 $\mathrm{NO}_{2}$ 浓度的差值: $\left[\mathrm{HO}_{2}\right]=\left(\left[\mathrm{NO}_{2}\right] \mathrm{amp}-\left[\mathrm{NO}_{2}\right] \mathrm{bas}\right) / \mathrm{CL}$. 有机 过氧自由基 $\mathrm{RO}_{2}$ 在同样条件下如能快速转变为 $\mathrm{HO}_{2}$ 的 话也可以被测定. 链长通过测定已知浓度的过氧自 由基而得到. 链长的大小取决于链传递与链终止反 应的相对速率, 通常在 50 300 之间.

近来有报道PERCA存在严重的水效应现象，即 水存在下CL会显著降低 ${ }^{[5,6]}$. 造成链长降低可能的原 因包括: (a) 链传递速率下降, (b) 链终止速率增加; (c) 自由基壁损耗加重 ${ }^{[5]}$. 实验发现(b)和(c)无法解释 水效应现象 ${ }^{[5]}$. Mihele等 ${ }^{[5]}$. 认为 $\mathrm{HO}_{2}$ 和 $\mathrm{H}_{2} \mathrm{O}$ 快速反应 形成的自由基-水加合物 $\mathrm{HO}_{2} \cdot \mathrm{H}_{2} \mathrm{O}$ 与 $\mathrm{NO}$ 反应生成了 $\mathrm{HNO}_{3}$ 或 $\mathrm{HOONO}$ 等分子产物可能是形成水效应的主 要原因:

$$
\mathrm{HO}_{2} \cdot \mathrm{H}_{2} \mathrm{O}+\mathrm{NO} \rightarrow \mathrm{HNO}_{3}+\mathrm{H}_{2} \mathrm{O}
$$




$$
\rightarrow \mathrm{HOONO}+\mathrm{H}_{2} \mathrm{O}
$$

(R4b)

然而, 至今没有(R4)的动力学数据, 因此上述假设一 直没有得到证实. 本文对水效应现象重新进行了实 验测定. 另外, 采用理论方法对(R4)的直接反应动力 学进行了研究. 在此基础上, 进一步通过模式计算模 拟水效应现象, 以对上述假设进行评估.

\section{1 实验部分}

\section{1 水效应的测定}

自由基通过管式流动技术动态发生. 图 1 是实验 装置示意图. 在 $184.9 \mathrm{~nm}$ 波段处光解空气中的水蒸气 产生等量的 $\mathrm{HO}_{2}$ 和 $\mathrm{OH}^{[7]}$ :

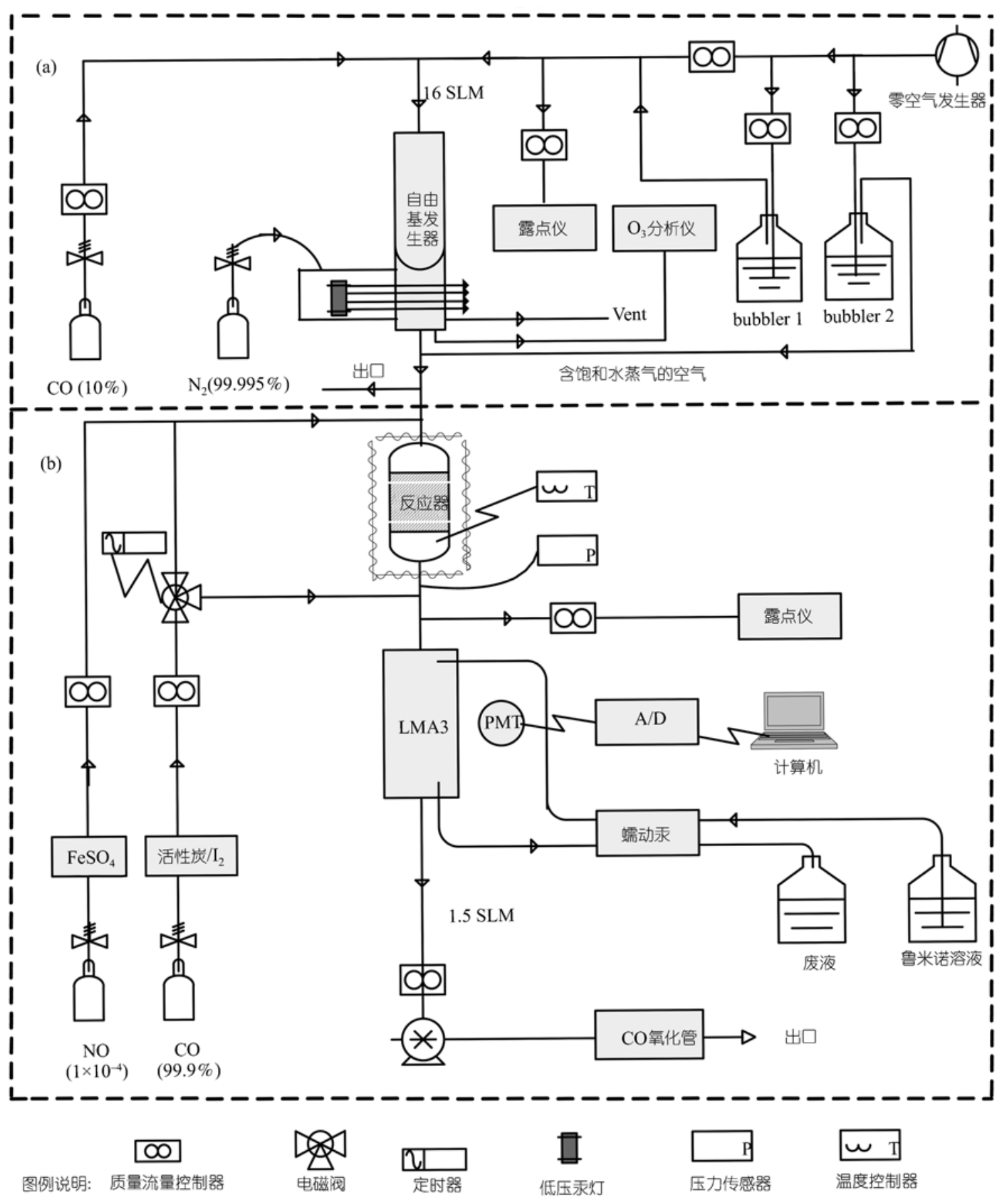

图 1 PERCA 水效应实验测定装置示意图

(a) 自由基发生器; (b) PERCA 系统 
$\mathrm{H}_{2} \mathrm{O}+h v(\lambda=184.9 \mathrm{~nm})+\mathrm{O}_{2}+\mathrm{M} \rightarrow \mathrm{HO}_{2}+\mathrm{OH}+\mathrm{M}(\mathrm{R} 5)$ 如在光解池中加入足量的 $\mathrm{CO}\left(\sim 1 \times 10^{-4}\right.$, 体积比浓度, 下同), 则 $\mathrm{OH}$ 被快速转变为 $\mathrm{HO}_{2}$. 这时自由基发生器 只产生 $\mathrm{HO}_{2} \cdot \mathrm{O}_{2}$ 在这一波段处也发生光解:

$$
\mathrm{O}_{2}+h v(\lambda=184.9 \mathrm{~nm})+\mathrm{O}_{2}+\mathrm{M} \rightarrow 2 \mathrm{O}_{3}+\mathrm{M}
$$

因此自由基发生器内同时有 $\mathrm{O}_{3}$ 产生. 生成的 $\mathrm{O}_{3}$ 用于 定量产生的自由基. 当气流处于层流状态时, 在光解 区域产生的 $\mathrm{HO}_{2}$ 浓度通过(F1)式确定 ${ }^{[7]}$ :

$$
\left[\mathrm{HO}_{2}\right]=\frac{\phi_{\mathrm{HO}_{2}} \sigma_{\mathrm{H}_{2} \mathrm{O}}}{\phi_{\mathrm{O}_{3}} \sigma_{\mathrm{O}_{2}}} \frac{\left[\mathrm{H}_{2} \mathrm{O}\right]}{\left[\mathrm{O}_{2}\right]}\left[\mathrm{O}_{3}\right] \text {. }
$$

$\mathrm{F}(1)$ 式中 $\phi_{\mathrm{HO}_{2}}$ 和 $\phi_{\mathrm{O}_{3}}$ 分别是 $\mathrm{HO}_{2}$ 和 $\mathrm{O}_{3}$ 的量子产率, 均 为 2 (当 $\mathrm{CO}$ 存在时). $\sigma_{\mathrm{H}_{2} \mathrm{O}}$ 和 $\sigma_{\mathrm{O}_{2}}$ 分别是 $\mathrm{H}_{2} \mathrm{O}$ 和 $\mathrm{O}_{2}$ 在 $184.9 \mathrm{~nm}$ 波段处的光吸收截面, 数值为 $7.1 \times 10^{-20[8]}$ 和 $1.2 \times 10^{-20} \mathrm{~cm}^{-2[9]}$.

光解在一石英管中 $(\phi 2.6 \mathrm{~mm} \times 50 \mathrm{~cm})$ 进行. 光源 是笔形低压 Hg灯 (penray lamp, UVE-5P, Sen Lights Corporation). 调节 $\mathrm{O}_{3}$ 在管中心与管内平均浓度的比 值为 $1: 2$, 则气体流动处于层流状态 ${ }^{[7]}$. 水经Bubbler 加入到自由基发生器中. 改变通过Bubbler气体的流 速可调节产生的自由基的浓度. 本实验发生的自由 基浓度为 $2 \times 10^{-11}$. 发生的自由基通过 Teflon管 $(\phi$ $0.159 \mathrm{~cm} \times 11.0 \mathrm{~cm}$ ) 进入PERCA进行测定.

自由基在被测定前, 样品需先与被水蒸气饱和 了的湿空气混合以改变样品的湿度. 由于混入了湿 空气，自由基浓度被稀释，需要另外测定同一条件下 加入等量干空气时反应的链长. 水效应通过干湿两 种条件下分别测得的链长 CLw 和 CLd 的比 $\gamma(\gamma=$ CLw/CLd 下同)表示. 化学放大在一 Pyrex 玻璃管 $(\phi 2 \mathrm{~mm} \times 16 \mathrm{~cm})$ 中进行. 管内 $\mathrm{NO}$ 和 $\mathrm{CO}$ 的浓度分别 为 $3.0 \times 10^{-6}$ 和 $10 \%$. CO 通过电磁阀 $(\mathrm{AV}-4345-11$, Electric Inc) 周期性地加入到反应器的入口和出口处. 电磁阀切换频率 (1 $\left.\mathrm{min}^{-1}\right)$ 用一时间继电器 (FT-011, Fine Inc.)进行控制. $\mathrm{NO}_{2}$ 采用鲁米诺化学发光仪 (LMA-3, Unisearch Associates Inc.) 进行测定. LMA-3 内置一抽气厡, 以 $1.5 \mathrm{~L} / \mathrm{min}$ 的流速将气体样品抽入 化学发光反应器内. 鲁米诺溶液(Luminol II, Unisearch Associates Inc.) 通过蠕动原 (SJ-1220, ATTO
Corporation) 以 $0.05 \mathrm{~mL} / \mathrm{min}$ 的流速输送到同一反应器 中. 每次实验前需对 LMA-3 进行校正. 标准 $\mathrm{NO}_{2}$ 气 体用 $\mathrm{O}_{3}$ 气相滴定过量 $\mathrm{NO}$ 的方法产生. 标准 $\mathrm{O}_{3}$ 气体 来自 $\mathrm{O}_{3}$ 校正仪(TECO, Model 49PS). 水蒸气浓度采 用冷镜式露点仪 (Yokogawa Electric Works, Model 2586)测定．臭氧浓度通过臭氧分析仪(TECO, Model 49C)测定. 所有气体的流速均采用质量流量控制器 (SEC-B40, STEC Inc.) 控制.

\section{2 理论计算}

在B3LYP/6-311G(2d, 2p) 水平下, 优化了(R4)路 径上反应物、中间体(IM)、过渡态(TS)和产物的几何 构型. IM和TS的真实性经振动分析及内禀反应坐标 (IRC) 计算进行了确认. 驻点能 (SPE) 进一步在 $\operatorname{CCSD}(\mathrm{T})$ 水平上进行了计算, 并进行了零点能 $(\mathrm{ZPE})$ 校正. 计算采用的程序是Gaussian $98^{[10]}$. 在IRC及SPE

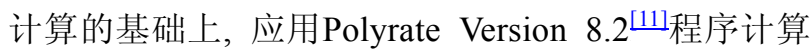
了(R4)主反应通道的速率常数.

\section{3 模式计算}

描述无水条件下自由基化学放大过程的化学模 式已有文献报道 ${ }^{[4]}$. 本文在文献工作基础上加入了 (R4a) 用以模拟水效应过程. 除(R4a)外, 模式中其他 反应的速率常数均来自文献[4]. 模式计算的初始条 件与实验相同. 反应物初始浓度分别为: $\left[\mathrm{HO}_{2}\right]=$ $2 \times 10^{-11},[\mathrm{NO}]=3 \times 10^{-6},[\mathrm{CO}]=10 \%$ 和 $\left[\mathrm{O}_{3}\right]=1.6 \times 10^{-9}$. $\mathrm{HO}_{2} \cdot \mathrm{H}_{2} \mathrm{O}$ 的初始浓度通过式 $\left[\mathrm{HO}_{2} \cdot \mathrm{H}_{2} \mathrm{O}\right]=K_{c}\left[\mathrm{HO}_{2}\right]$ $\left[\mathrm{H}_{2} \mathrm{O}\right]$ 计算, 式中 $K_{c}$ 表示反应 $\mathrm{HO}_{2}+\mathrm{H}_{2} \mathrm{O} \rightarrow \mathrm{HO}_{2} \cdot \mathrm{H}_{2} \mathrm{O}$ 的平衡常数, 其数值来自文献[12]. 计算中温度和压 力分别设定为 $298 \mathrm{~K}$ 和 $1 \times 10^{5} \mathrm{~Pa}$. 模式计算采用 VBA 与Matlab混合技术编写的程序进行. 该程序还可以进 行误差分析. 误差分析采用蒙特卡罗算法估计从输 入变量误差到计算结果的传递.

\section{2 结果与讨论}

\section{1 水效应的测定}

图 2 是在室温 $(298 \pm 2) \mathrm{K}$ 及大气压 $\left(1 \times 10^{5} \mathrm{~Pa}\right)$ 下得 
到的PERCA水效应曲线. 实验结果表明, $\gamma$ 随水浓度 呈指数衰减. 这与文献 $[5,6]$ 报道的结果一致. 以往水 效应研究中没有考虑自由基从自由基发生器到自由 基测定之间输运过程中可能的损耗. 为此, 本文另外 测定了 $\mathrm{HO}_{2}$ 与 $\mathrm{OH}$ 混合自由基的水效应. 因为PERC A 测定 $\mathrm{HO}_{2}$ 和 $\mathrm{OH}$ 的灵敏度相同 ${ }^{[4]}$, 但 $\mathrm{OH}$ 的壁反应速率 常数显著小于 $\mathrm{HO}_{2}{ }^{[5]}$. 然而, 从图 2 可以看出, 水效应 随自由基的类型无显著变化, 表明自由基在输运过 程中的损耗可以忽略.

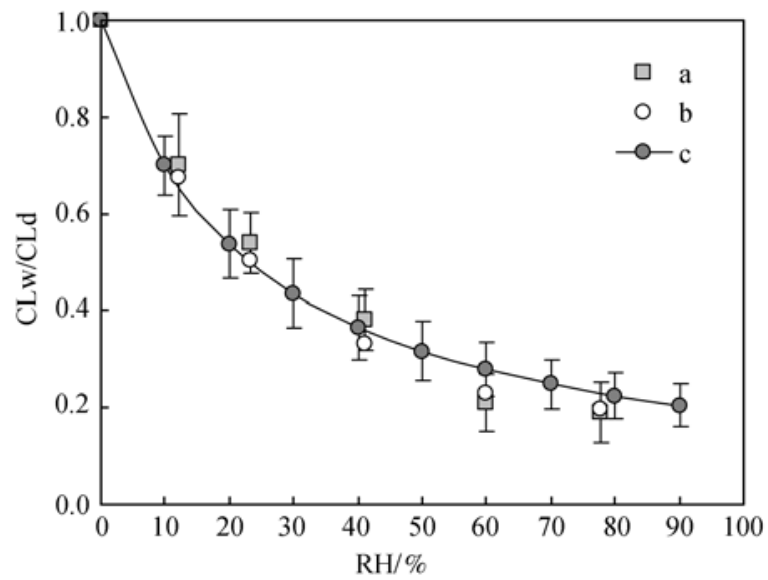

图 2 在室温 $(298 \pm 2) \mathrm{K}$ 及大气压 $\left(1 \times 10^{5} \mathrm{~Pa}\right)$ 下得到的 PERCA 水效应曲线

$a$ : 采用 $\mathrm{HO}_{2}$ 测得的水效应曲线; $b$ : 采用 $\mathrm{HO}_{2}+\mathrm{OH}$ 测得的水效应曲 线; $\mathrm{c}$ : 模式计算得到的水效应曲线. $\left[\mathrm{HO}_{2}\right]=2 \times 10^{-11},\left[\mathrm{HO}_{2}\right]+[\mathrm{OH}]=$ $2 \times 10^{-11}$

\section{2 理论计算}

图 3 是 $B 3 \mathrm{LYP} / 6-311 \mathrm{G}(\mathrm{d}, \mathrm{p})$ 水平下优化得到的 (R4)反应路径上各驻点的几何构型. 各驻点对应物种 的总能量及经零点振动能校正后的相对能量列在表 1. 图 4 是反应势能剖面示意图. 从图 4 可以看出反应 存在多个中间体和反应通道. 中间体IM1, IM1', IM2, IM2', IM3, IM3'和IM4 分别经过相应的过渡态到达产 物 $\mathrm{HNO}_{3}+\mathrm{H}_{2} \mathrm{O}$. 通道IM1 $\rightarrow \mathrm{TS} 1 \rightarrow \mathrm{HNO}_{3}+\mathrm{H}_{2} \mathrm{O}$ 的势垒 最低, 为 $125.1 \mathrm{~kJ} / \mathrm{mol}$. 对比反应 $\mathrm{HO}_{2}+\mathrm{NO} \rightarrow \mathrm{HNO}_{3}$ 的势垒 $163.0 \mathrm{~kJ} / \mathrm{mol}^{[13]}$, 可以看出水的存在起到了降
低反应势垒的作用. 中间体 IM5, IM6 和 IM7 分别经 过相应的过渡态到达产物 $\mathrm{HOONO}+\mathrm{H}_{2} \mathrm{O}$, 其最低反 应通道是 $\mathrm{IM} 6 \rightarrow \mathrm{TS} 6 \rightarrow \mathrm{HOONO}+\mathrm{H}_{2} \mathrm{O}$ ，反应势垒为 $169.7 \mathrm{~kJ} / \mathrm{mol}$, 是次要反应通道.

在能量及反应势能面计算的基础上，根据传统 过渡态理论(TST) 和变分过渡态理论(CVT), 分别计 算了主反应通道在 200 2000 K 温度范围内的速率常 数 $k^{\mathrm{TST}}$ 和 $k^{\mathrm{CVT}}$, 并应用小曲率隧道效应模型 $(\mathrm{SCT})$ 对 $k^{\mathrm{CVT}}$ 进行了校正，得到了经小曲率隧道效应校正后的 速率常数 $k^{\mathrm{CVT} / \mathrm{SCT}}$, 结果见图 4. 由图 4 可以看出 $k^{\mathrm{TST}}$ 和 $k^{\mathrm{CVT}}$ 在计算温度范围内无显著差异, 表明在这一 温度范围内变分作用对速率常数的影响不大. $k^{\mathrm{CVT}}$ 与 $k^{\mathrm{CVT} / \mathrm{SCT}}$ 在低温段明显不同, 但当温度大于 $1000 \mathrm{~K}$ 时, 两曲线几乎重合, 说明在低温区速率常数须经量子 力学隧道效应校正. 在 200 2000 K 温度范围内, 拟 合得到 $k^{\mathrm{CVT} / \mathrm{SCT}}$ 的三参数 Arrhenius 方程为: $k^{\mathrm{CVT} / \mathrm{SCT}}$ $=5.49 \times 10^{7} T^{1.03} \exp (-14798 / T)$.

\section{3 水效应的数值模拟}

模式计算得到的水效应曲线也列在图 2 中. 模式 计算结果中的误差线来自(R4a)速率常数的计算误差 (假定为 $20 \%$ ). 可以看出在误差范围内，模拟和实验 测定得到的水效应曲线基本一致，表明文献[4]提出 的水效应的假设可以解释水效应现象.

\section{3 结论}

测定了 $\mathrm{HO}_{2}$ 及 $\mathrm{HO}_{2}+\mathrm{OH}$ 混合自由基化学放大过 程中的水效应，没有发现水效应随自由基的类型有 显著变化，表明自由基在采样过程中的损耗可以忽 略. $\mathrm{HO}_{2} \cdot \mathrm{H}_{2} \mathrm{O}$ 与 $\mathrm{NO}$ 反应主要生成分子产物 $\mathrm{HNO}_{3}+\mathrm{H}_{2} \mathrm{O}$, 导致链传递速率降低. 在 200 2000 K 温 度范围内，拟合得到经小曲率隧道效应模型校正后 的三参数 Arrhenius 方程为: $k^{\mathrm{CVT} / \mathrm{SCT}}=5.49 \times 10^{7} T^{1.03}$ $\exp (-14798 / T)$. 模式计算得到的水效应曲线与实验 测定的基本一致，表明(R4a)可能是产生水效应的主 要原因。 


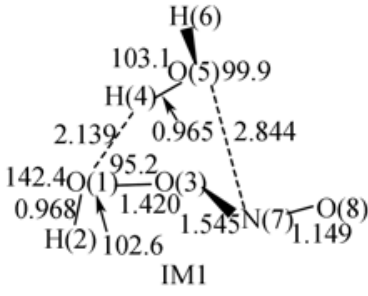

IMl

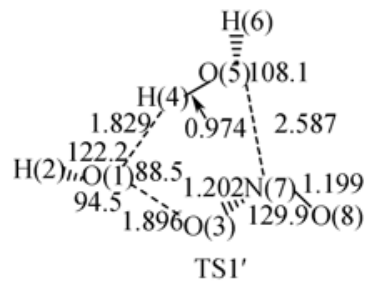

TS1'

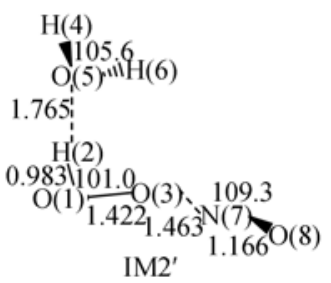

H(2) $113.22-\mathrm{H}(4), 108.2 \mathrm{H}(6)$

2.212

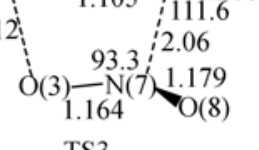

TS3

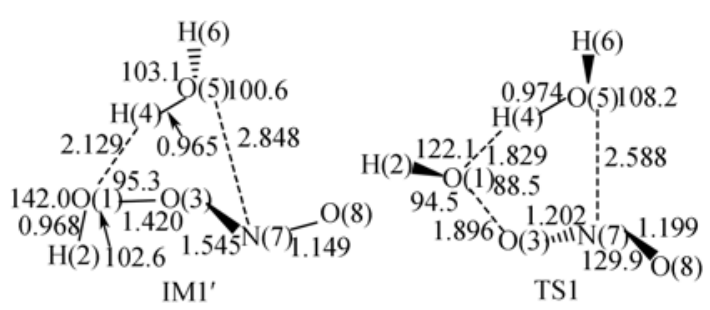

$\mathrm{IM1}^{\prime}$

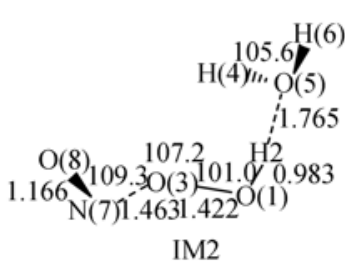

IM2

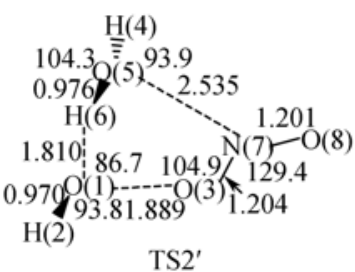

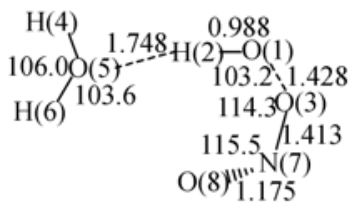

IM3

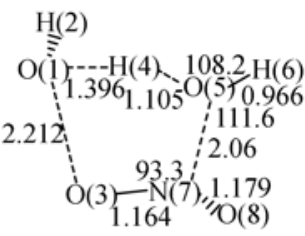

TS3'

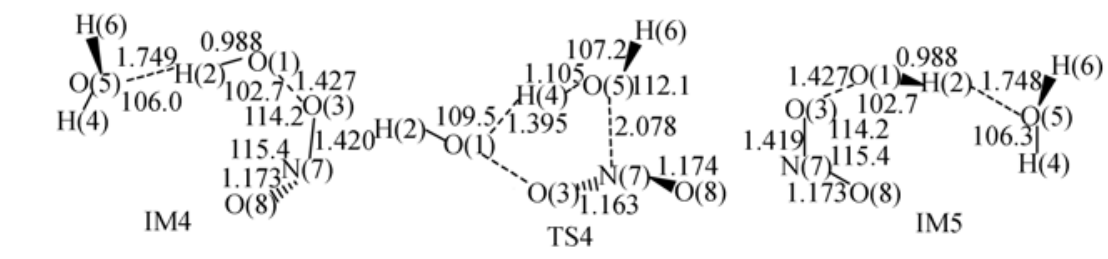

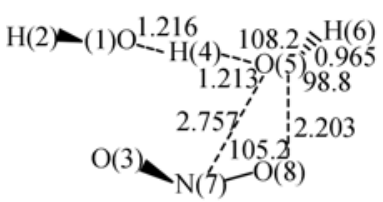

TS5

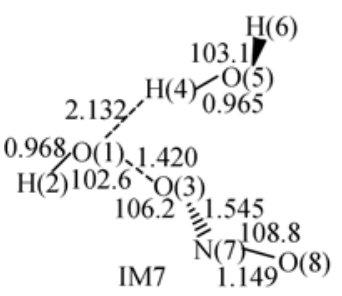

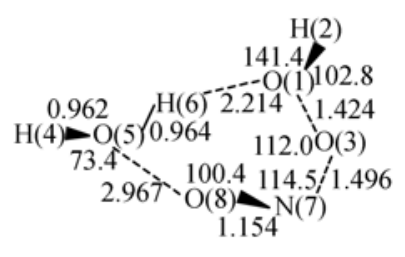

IM6

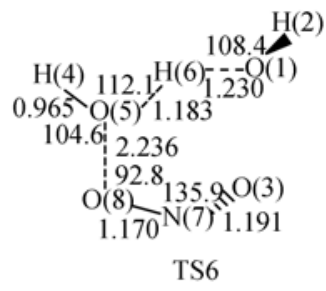

TS6

图 3 B3LYP/6-311G $(d, p)$ 水平下优化得到的各驻点的几何构型及其参数 键长单位: $0.1 \mathrm{~nm}$, 键角单位: $\left(^{\circ}\right)$ 

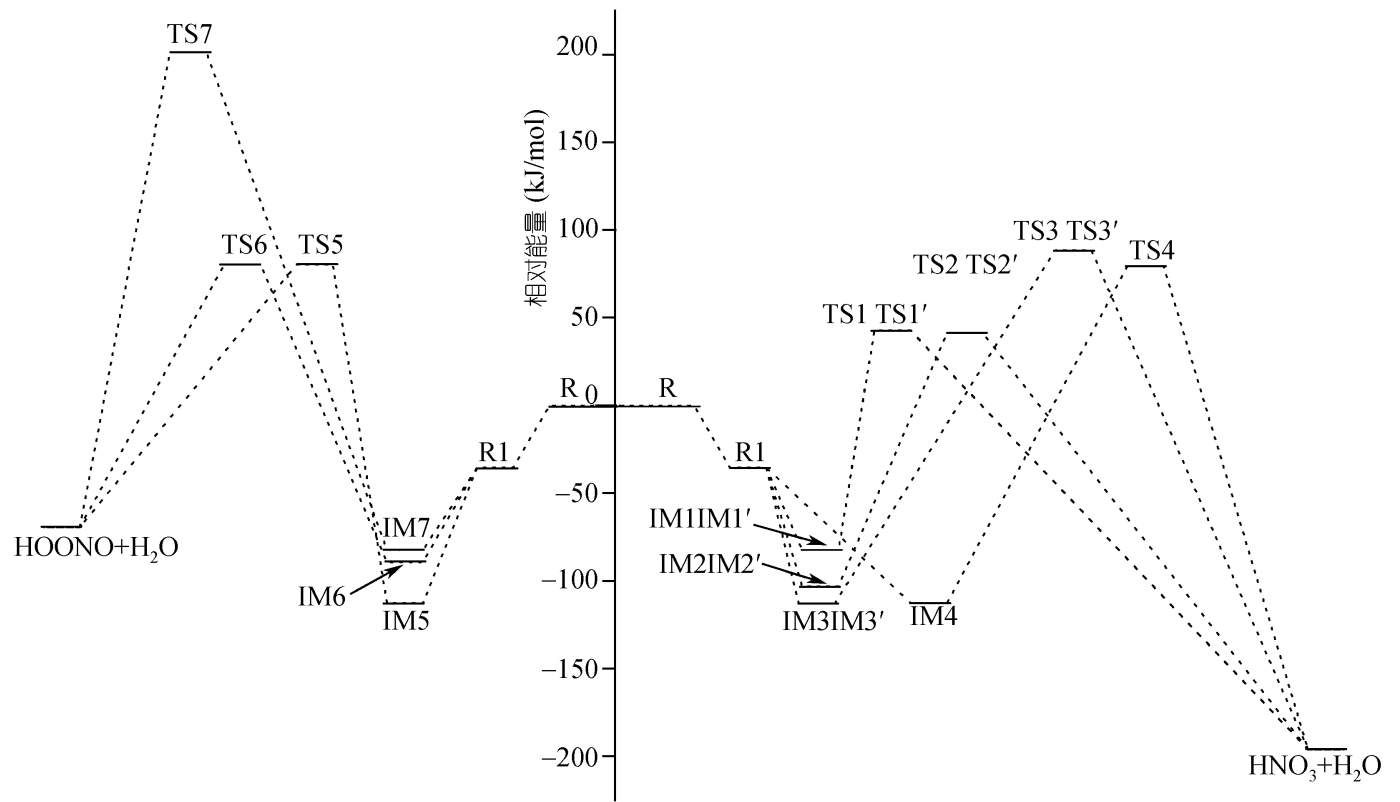

图 $4 \mathrm{CCSD}(\mathrm{T}) / / \mathrm{B} 3 \mathrm{LYP} / 6-311 \mathrm{G}(\mathrm{d}, \mathrm{p})$ 水平下计算得到的反应 $\mathrm{HO}_{2} \cdot \mathrm{H}_{2} \mathrm{O}+\mathrm{NO}$ 的势能面

表 1 不同理论水平下计算得到的 $\mathrm{HO}_{2} \cdot \mathrm{H}_{2} \mathrm{O}+\mathrm{NO}$ 反应路径上各驻点总能量及零点能 (hatree/particle)

\begin{tabular}{|c|c|c|c|c|}
\hline Species & B3LYP & ZPE & $\operatorname{CCSD}(\mathrm{T})$ & $\operatorname{CCSD}(\mathrm{T})+\mathrm{ZPE}$ \\
\hline $\mathrm{HO}_{2}+\mathrm{NO}+\mathrm{H}_{2} \mathrm{O}$ & -357.284577 & 0.039981 & -356.530771 & -356.490790 \\
\hline $\mathrm{H}_{2} \mathrm{O} \cdot \mathrm{HO}_{2}+\mathrm{NO}$ & -357.300779 & 0.044769 & -356.549015 & -356.504246 \\
\hline IM1 & -357.318949 & 0.046972 & -356.569120 & -356.522148 \\
\hline $\mathrm{IM}^{\prime}$ & -357.318931 & 0.046992 & -356.569112 & -356.522120 \\
\hline TS1 & -357.263994 & 0.045767 & -356.520280 & -356.474513 \\
\hline TS1' & -357.263992 & 0.045769 & -356.520291 & -356.474522 \\
\hline IM2 & -357.328076 & 0.047643 & -356.577620 & -356.529977 \\
\hline IM2' & -357.328081 & 0.047638 & -356.577620 & -356.529982 \\
\hline TS2 & -357.266461 & 0.046118 & -356.521163 & -356.475045 \\
\hline TS2' & -357.266462 & 0.046118 & -356.521165 & -356.475047 \\
\hline IM3 & -357.332750 & 0.047849 & -356.581590 & -356.533741 \\
\hline IM3' & -357.332761 & 0.047839 & -356.581599 & -356.533760 \\
\hline TS3 & -357.269248 & 0.044586 & -356.501643 & -356.457057 \\
\hline TS3' & -357.269246 & 0.044588 & -356.501675 & -356.457087 \\
\hline IM4 & -357.332647 & 0.047880 & -356.581576 & -356.533696 \\
\hline TS4 & -357.273942 & 0.044908 & -356.505364 & -356.460456 \\
\hline IM5 & -357.332655 & 0.047871 & -356.581572 & -356.533701 \\
\hline TS5 & -357.275966 & 0.042762 & -356.502938 & -356.460176 \\
\hline IM6 & -357.321596 & 0.046511 & -356.571299 & -356.524788 \\
\hline TS6 & -353.272382 & 0.042384 & -356.502533 & -356.460149 \\
\hline IM7 & -357.318943 & 0.046980 & -356.569114 & -356.522134 \\
\hline TS7 & -357.219471 & 0.042802 & -356.456780 & -356.413978 \\
\hline $\mathrm{HNO}_{3}+\mathrm{H}_{2} \mathrm{O}$ & -357.367040 & 0.047781 & -356.613181 & -356.565400 \\
\hline $\mathrm{HOONO}+\mathrm{H}_{2} \mathrm{O}$ & -357.313930 & 0.044395 & -356.561632 & -356.517237 \\
\hline
\end{tabular}




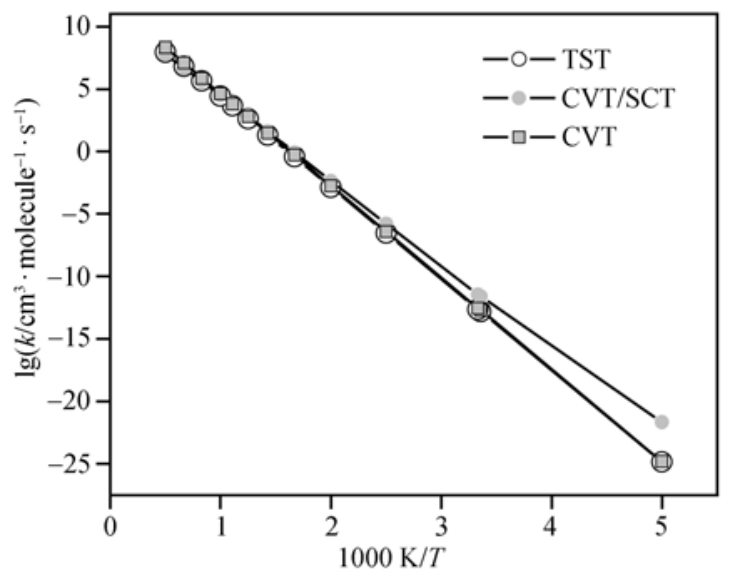

图 $5200 \sim 2000 \mathrm{~K}$ 温度范围内 $(\mathrm{R} 4 \mathrm{a})$ 主反应通道速率常数随温度的变化

致谢作者感谢明尼苏达大学化学系与超级计算机研究所提供的 Polyrate 程序.

\section{参考文献}

1 Fleming Z L, Monks P S, Rickard A R, Heard D E, Bloss W J, Seakins P W, Still T J, Sommariva R, Pilling M J, Morgan R, Green T J, Brough N, Mills G P, Penkett S A, Lewis A C, Lee J D, Saiz-Lopez A, Plane J M C. Peroxy radical chemistry and the control of ozone photochemistry at Mace Head, Ireland during the summer of 2002. Atmos Chem Phys, 2006, 6: 2193-2214

2 Qi B, Takami A, Hatakeyama S. Peroxy radical concentrations measured at a forest canopy in Nikko, Japan, in Summer 2002. J Atmos Chem, 2005, 52: 63-79 [DOI]

3 Cantrell C A, Stedman D H. A possible technique for the measurement of atmospheric peroxy radicals. Geophys Res Lett, 1982, 9: $846-849$

4 Hastie D R, Weissenmayer M, Burrows J P, Harris G W. Calibrated chemical amplifier for atmospheric RO $\mathrm{R}_{x}$ measurements. Anal Chem, 1991, 63: 2048-2057[DOI]

5 Mihele C M, Hastie D R. The sensitivity of the radical amplifier to ambient water vapour. Geophys Res Lett, 1998, 25(11): 19111913 [DOI]

6 Reichert L, Andres Hernandez M D, Stobener D, Burkert J, Burrows J P. Investigation of the effect of water complexes in the determination of peroxy radical ambient concentrations: Implications for the atmosphere. J Geophys Res, 2003, 108: 4017-4032 [DOI]

7 Schultz M, Heitlinger M, Mihelcic D, Volz-Thomas A. Calibration source for peroxy radicals with built-in actinometry using $\mathrm{H}_{2} \mathrm{O}$ and $\mathrm{O}_{2}$ photolysis at $185 \mathrm{~nm}$. J Geophys Res, 1995, 100: 18811-18816[DOI]

8 Cantrell C A, Zimmer A, Tyndall G S. Absorption cross-sections for water vapor from $183 \mathrm{~nm}$ to $193 \mathrm{~nm}$. Geophys Res Lett, 1997, 24: $2195-2198 \underline{[\mathrm{DO}]}$

9 Hofzumahaus A, Brauers T, Aschmutat U, Brandenburger U, Dorn H P, Hausmann M, Hesling F, Holland F, Plass-Dulmer C, Sedlacek M, Weber M, Ehhalt D H. Reply. J Geophys Res, 1997, 24: 3039-3040

10 Frisch M J, Trucks G W, Schlegel H B, Scuseria G E, Robb M A, Cheeseman J R, Zakrzewski V, G, Montgomery Jr J A, Stratmann R E, Burant J C, Dapprich S, Millam J M, Daniels A D, Kudin K N, Strain M C, Farkas Ö, Tomasi J, Barone V, Cossi M, Cammi R, Mennucci B, Pomelli C, Adamo C, Clifford S, Ochterski J, Petersson G A, Ayala P Y, Cui Q, Morokuma K, Salvador P, Dannenberg J J, Malick D K, Rabuck A D, Raghavachari K, Foresman J B, Cioslowski J, Ortiz J V, Baboul A G, Stefanov B B, Liu G, Liashenko A, Piskorz P, Komáromi I, Gomperts R, Martin R L, Fox D J, Keith T, Al-Laham M A, Peng C Y, Nanayakkara A, Challacombe M, Gill P M W, Johnson B, Chen W, Wong M W, Andres J L, Gonzalez C, Head-Gordon M, Replogle E S, Pople J A. Gaussian 98, revision A.9: Gaussian, Inc [CP]. Pittsburgh, PA, 1998

11 Chuang Y Y, Corchado J C, Fast P L, Villà J, Hu W P, Liu Y P, Lynch G C, Jackels C F, Nguyen K A, Gu M Z, Rossi I, Coitiño E L, Clayton S, Melissas V S. Polyrate-version 8.2, Minneapolis [CP]. University of Minnesota, 1999

12 Aloisio S, Francisco J S. Experimental Evidence for the Existence of the $\mathrm{HO}_{2}-\mathrm{H}_{2} \mathrm{O}$ Complex. J Phys Chem A, 2000, 104: 6597-6601 [DOI]

13 Sumath R, Peyerimhoff S D. An abinito molecular orbital study of the potential energy surface of the $\mathrm{HO}_{2}+\mathrm{NO}$ reaction. J Chem Phys, 1997, 107: 1972-1879 